\title{
MAGNETIC BRAKING AND THE OBLIQUE ROTATOR MODEL
}

\author{
L. MESTEL and C. S. SELLEY
}

The University of Manchester, Manchester, England

This work investigates the dynamical evolution of a rotating magnetic star which drives a stellar wind. The basic magnetic field of the star is supposed symmetric about an axis, which is inclined at an angle $\chi$ to the rotation axis $\mathbf{k}$ (Figure 1). We adopt the familiar equations of an inviscid perfectly conducting gas. In a steady state, the velocity as seen in a frame rotating with the star is taken as

$$
\mathbf{v}=\kappa \mathbf{H}
$$

where $\kappa$ is a scalar. In the inertial frame, the velocity $\mathbf{v}$ is no longer parallel to $\mathbf{H}$, but has the velocity of corotation superposed.

Close to the star, the strong magnetic field controls the flow of the wind, in particular maintaining approximate corotation with the star. The required torque on the gas

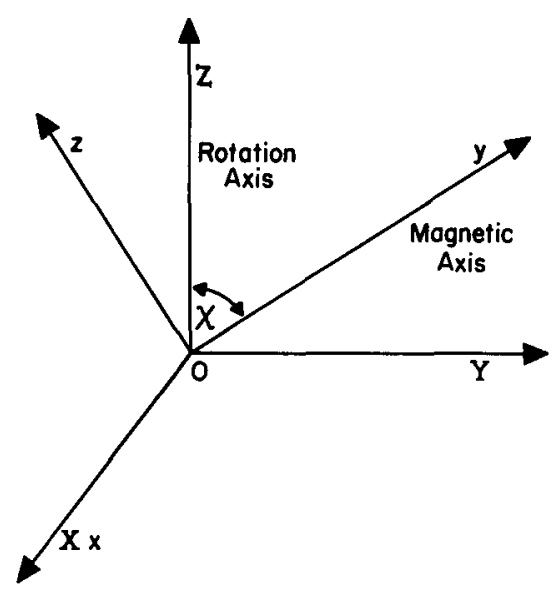

Fig. 1. Coordinate axes describing the stellar rotation and magnetic field.

arises from a perturbation $\mathbf{H}^{\prime}$ to the basic magnetic field $\overline{\mathbf{H}}$. When $\chi=0, \mathbf{H}^{\prime}$ is a twist about the rotation axis. By (1), the gas must have an azimuthal component of velocity in the rotating frame, but close to the star this will be small compared with the rotational velocity of the frame. Further out the lag becomes more and more pronounced. At great distances from the star, corotation ceases entirely: the field exerts effectively no torques on the gas, which therefore flows with approximate conservation of angular momentum, drawing out the field into a spiral configuration (Parker, 1958). The change-over occurs near the critical surface $S_{A}$ at which the wind-speed in the inertial frame equals the speed of Alfvén waves along the poloidal field. 
In the absence of a magnetic field, the star experiences a slight braking torque, parallel to the rotation axis, due to transport by the stellar wind of the angular momentum it had at the stellar surface. When $\chi=0$ or $\pi / 2$ the principal effect of the magnetically enforced corotation is to increase greatly the angular momentum flow per unit mass loss. For general $\chi$, the reduction in symmetry yields an additional component of torque in the direction $O Y$ perpendicular to the rotation axis $O Z$ (Figure 1). With the field frozen into the star, the principal effect of this $Y$-torque is to cause the instantaneous axis of rotation to precess about $O X$, changing the angle $\chi$; simultaneously the magnetic axis rotates steadily in space. If the $Y$-torque is negative (like the $Z$-torque), then $\dot{\chi}>0$; if positive, $\dot{\chi}<0$ (Mestel, 1968b). The problem is at once seen to be relevant to the oblique rotator model.

The axisymmetric case has been extensively studied, and the results extrapolated to apply to the sun. The magnetic field is conveniently resolved into a poloidal part, present if the star is non-rotating, and the toroidal perturbation $\mathbf{H}^{\prime}$ due to the rotation. The toroidal equation of motion has a simple integral, from which $\mathbf{H}^{\prime}$ can be determined. This integral is easily interpreted: there is no interchange of the $\mathbf{k}$-component of angular momentum between adjacent field-streamlines, but there is a steady flow of k-angular momentum along the field-streamlines, carried jointly by the gas and the magnetic tensions (Lüst and Schlüter, 1955). It turns out that the total torque on the star is given by assuming that the gas is kept in a state of strict corotation out to the Alfvén surface $S_{A}$, even though the actual rotation of the gas at $S_{A}$ is well below the stellar rotation (Mestel, 1966, 1967, 1968a; Weber and Davis, 1967; Modisette, 1967; Ferraro and Bhatia, 1967).

With general $\chi$ there is no simple integral relating the perturbed and unperturbed fields, and k-angular momentum will be interchanged between adjacent field-lines. Analogues of the axisymmetric poloidal and toroidal fields - called $\overline{\mathbf{H}}$ and $\mathbf{H}^{\prime}$ - can be conveniently defined in terms of their properties under reflection in the plane $O Y Z$ in Figure 1. The actual method of solution is by a perturbation procedure in the stellar rotation $\alpha$, the term of order $\alpha$ being the first approximation to $\mathbf{H}^{\prime}$. Terms of the second order in the equation include the centrifugal force, and the magnetic force term $\left(\nabla \times \mathbf{H}^{\prime}\right) \times \mathbf{H}^{\prime} / 4 \pi$, and they will modify the poloidal field (seriously if the rotation is rapid); however, we have not as yet gone beyond first-order terms. Once $\mathbf{H}^{\prime}$ is known, the $Y$ - and $Z$-components of torque can be computed directly by integrating the moment of the Maxwell stresses over the stellar surface. Before performing this expansion, however, we derive an exact transform for the torque $\mathbf{L}$ on the star. It can be written as the sum of three integrals:

$$
\begin{aligned}
-\mathbf{L}=(\alpha \mathbf{k} \times \mathbf{h}) & +\int_{S_{A}}\left(P+\frac{\mathbf{H}^{2}}{8 \pi}\right)(\mathbf{r} \times \mathbf{n}) \mathrm{d} S \\
& +\int_{S_{A}} \varrho(\mathbf{v} \cdot \mathbf{n})(\mathbf{r} \times(\alpha \mathbf{k} \times \mathbf{r})) \mathrm{d} S,
\end{aligned}
$$


where

$$
\mathbf{h}=\int_{\tau} \mathbf{r} \times \varrho(\mathbf{v}+\alpha \mathbf{k} \times \mathbf{r}) \mathrm{d} \tau
$$

and $\tau$ is the volume between the star and the Alfvénic surface $S_{A}$. The vector $\mathbf{h}$ is the angular momentum of the gas flowing through $\tau$, and the first term on the right of (2) gives the torque that must be exerted to rotate this 'flywheel' at the rate $\alpha \mathbf{k}$. The integrated moment of the total pressure $\left(P+H^{2} / 8 \pi\right)$ would vanish if the Alfvénic surface were spherical. The last term is seen to be the generalization of the axisymmetric result quoted above; and in fact if the magnetic field is axisymmetric, and $\chi=0$, the first two terms in (2) vanish by symmetry.

The simplest possible poloidal field is the split monopole, with the field-lines radial and the field-strength independent of angle, and with a sheet of gas across the cut keeping the oppositely-directed field-lines from diffusing into each other. It is easily shown that the first-order perturbation in this field due to rotation is again just a twist about the rotation axis, with an associated distortion in the cut. The field still has enough symmetry for the net torque to be parallel to the rotation axis. The Alfvénic surface is a sphere, and the first-order perturbation $\left(P^{\prime}+\overline{\mathbf{H}} \cdot \mathbf{H}^{\prime} / 4 \pi\right)$ in the total pressure is zero.

We now suppose the flux distribution over the stellar surface departs from that of a split monopole by a small part of order $\varepsilon$, which is expanded in surface harmonics. In addition to the $Z$-torque - of order 2 - there is now a $Y$-component with a dominant part of order $\alpha \varepsilon$. Because of the properties of the split monopole solution, only the flow surface integral in (2) contributes to this order, with the velocity field $\mathbf{V}$ and the distortion of the Alfvénic surface $S_{A}$ computed to zero order in $\alpha$ but to the first order in $\varepsilon$. Thus we have a result analogous to the axisymmetric result: the transform (2) enables us to compute the torque to order $\alpha \varepsilon$ without a prior computation of the magnetic field perturbation of this order.

It is convenient to write the flux distribution over the stellar surface $R$ in the form

$$
\frac{\bar{H}_{1 r}(R)}{\bar{H}_{0}(R)}=\varepsilon \sum Y_{n}\left(\theta_{1} \lambda\right)
$$

where $\bar{H}_{0}$ is the basic (split monopole) field, and

$$
\begin{aligned}
Y_{n}\left(\theta_{1} \lambda\right)= & a_{0}(n) P_{n}(\cos \theta) \\
& +\sum_{k=1}^{n}\left\{a_{k}(n) \cos k \lambda+b_{k}(n) \sin k \lambda\right\} P_{n}^{k}(\cos \theta)
\end{aligned}
$$

in standard notation. We then find that the $Y$ and $Z$ components of the torque $\mathbf{L}$ are related by

$$
\frac{L_{Y}}{L_{Z}}=-\varepsilon A\left(\frac{r_{c}}{r_{A}}\right)\left\{\left[\frac{1}{2} a_{0}(2)-a_{2}(2)\right] \sin 2 \chi-a_{1}(2) \cos 2 \chi\right\}
$$


where the coefficient $A\left(r_{c} / r_{A}\right)$ depends on the ratio of the zero-order Alfvénic and Sonic spheres. For the parameter range in which $A$ is not so small that the effect is negligible, $A$ is positive and about 0.04 . The maximum change in $\chi$ is then found to be of order

$$
|\Delta \chi| \simeq .04 \varepsilon \log \left\{(C \alpha)_{i} /(c \alpha)_{f}\right\}
$$

where $C$ is the moment of inertia of the star, and the suffixes $i$ and $f$ refer respectively to an initial state (e.g. on the Hayashi track), and to a final (main-sequence) state. Thus for the field structure studied $|\Delta \chi|$ is not large. However, the perturbed split monopole field was adopted purely for mathematical convenience. A more realistic basic field structure will allow the field to be curl-free near the star, with the wind channelled parallel to the strong field rather than with the field lines pulled out by the wind; for such structures $\left|L_{Y} / L_{Z}\right|$ and so also $\Delta \chi$ will be much larger.

The sign of $\chi$ depends on $\chi$ and on the flux distribution over the stellar surface, as measured by the coefficients $a_{k}(2)$. If $a_{1}(2)=0$ i.e. the $Y_{2}$ part of $\bar{H}_{1}$ is antisymmetric in the equator - then $\chi$ will steadily approach 0 or $\pi / 2$, depending on whether $\left[\frac{1}{2} a_{0}(2)-a_{2}(2)\right] \gtrless 0$ respectively. If $a_{1}(2) \neq 0$ the same criterion decides whether $\chi$ approaches a small or large value. We expect that with the more realistic approximately curl-free structure, the sign of $\chi$ will still be largely determined by the photospheric flux distribution.

The present work suggests the possibility that the same magnetic coupling with a stellar wind that may explain the slow rotations of the magnetic stars may also explain the large inclinations of the magnetic and rotation axes required by the oblique rotator model. It is hoped to extend the work in several ways. We need to compute the torques for more plausible field structures. One can ask whether those surface flux distributions that do yield large asymptotic values are also consistent with observed Zeeman shifts and cross-over effects. There is the formidable problem in internal stellar hydromagnetics of explaining the required flux distribution. Finally, we note that any dissipation of kinetic energy of stars (at fixed angular momentum) will tend to reduce the angle $\chi$ (Spitzer, 1958); the time-scale of this process should be estimated and compared with that for the present process.

\section{References}

Deutsch, A. J.: 1958, in Electromagnetic Processes in Cosmical Physics (ed. by B. Lehnert), Cambridge University Press, Cambridge, England.

Ferraro, V. C. A. and Bhatia, V. B.: 1967, Astrophys. J. 147, 220.

Lüst, R. and Schlüter, A.: 1955, Z. Astrophys. 38, 190.

Mestel, L.: 1966, Liège Symposium.

Mestel, L.: 1967, in Stellar Magnetism in Plasma Astrophysics, 39th Enrico Fermi School, Varenna, 1966.

Mestel, L.: 1968a, Monthly Notices Roy. Astron. Soc. 138, 359.

Mestel, L.: 1968b, Monthly Notices Roy. Astron. Soc. 140, 177.

Modisette, J. L.: 1967, J. Geophys, R. 72, 1521.

Parker, E. N.: 1958, Astrophys. J. 128, 664. 
Spitzer, Jr., L.: 1958, in Electromagnetic Processes in Cosmical Physics (ed. by B. Lehnert), Cambridge University Press, Cambridge, England.

Weber, E. J. and Davis Jr., L.: 1967, Astrophys. J. 148, 271.

\section{Discussion}

Deutsch: The A stars are not known to support stellar winds, of course. One can argue that the magnetic A stars are the least likely of any stars to support winds.

Selley: Yes, on the main sequence the early-type stars lack the violent surface convection zones necessary for the generation of an expanding corona. We are postulating however, that magnetic braking is operative during the Hayashi phase. We suppose that the magnetic field is primeval and while it is sufficiently strong to resist continuous deformation by the Hayashi turbulence it does not suppress convection. In the Hayashi phase while we expect all A-type stars to possess a dynamo-built surface field, a magnetic A star will experience far greater magnetic braking if it has, in addition, a primeval magnetic field.

The Ap stars are abnormally slow rotators; at the same time many of them show periodic field reversal which indicates oblique rotators with large values of the angle $\chi$.

Magnetic braking on the Hayashi track due to a primeval magnetic field explains both the slow rotation and the postulated high angles of obliquity of these Ap stars. It remains to be seen whether the same result is given by more realistic models of the magnetic fields.

Nariai: It may be possible that the rotation of a star with a strong magnetic field is decelerated during a part of the Hayashi contraction phase when the star has a convection zone and corona, it then becoming a slowly rotating peculiar A star in the main-sequence phase. 